\title{
Controlled Passage through Resonance for Flexible Vibration Units
}

\author{
Dmitry A. Tomchin, ${ }^{1}$ Olga P. Tomchina, ${ }^{2}$ and Alexander L. Fradkov ${ }^{3}$ \\ ${ }^{1}$ Institute of Problems in Mechanical Engineering, 61 Bolshoy Avenue V.O., Saint Petersburg 199178, Russia \\ ${ }^{2}$ Saint Petersburg State University of Architecture and Civil Engineering (SPSUACE), 2nd Krasnoarmeiskaya Street 4, \\ Saint Petersburg 190005, Russia \\ ${ }^{3}$ University ITMO, St. Petersburg State University and Institute of Problems in Mechanical Engineering, \\ 61 Bolshoy Avenue V.O., Saint Petersburg 199178, Russia
}

Correspondence should be addressed to Alexander L. Fradkov; fradkov@mail.ru

Received 28 December 2014; Revised 24 March 2015; Accepted 24 March 2015

Academic Editor: Tomasz Kapitaniak

Copyright (C) 2015 Dmitry A. Tomchin et al. This is an open access article distributed under the Creative Commons Attribution License, which permits unrestricted use, distribution, and reproduction in any medium, provided the original work is properly cited.

The problem of controlled passage through resonance zone for mechanical systems with several degrees of freedom is studied. Control algorithm design is based on speed-gradient method and estimate for the frequency of the slow motion near resonance (Blekhman frequency). The simulation results for two-rotor flexible vibration units illustrating efficiency of the proposed algorithms and fractal dependence of the passage time on the initial conditions are presented. The novelty of the results is in demonstration of good behavior of the closed loop system if flexibility is taken into account.

\section{Introduction}

A typical problem for control of vibration units is passing through resonance at startup mode of vibroactuators, in the case of the operating modes belonging to a postresonance zone. Such a problem arises in the case when the power of a motor is not sufficient for passage through resonance zone due to Sommerfeld effect [1]. Dynamics of near resonance behavior are nonlinear and very complicated. Their analysis attracts attention of researchers for about 50 years [2-4].

Perhaps the first approach to the problem of controlled passage through resonance zone was the so-called "double start" method due to Gortinskii et al. [5]. The method is based on the insertion of time relay into motor control circuit for repeatedly switching on and off motor at precalculated time instants. Basically, this and other feedforward (nonfeedback) methods are characterized by difficulties in calculation of switching instants of a motor and sensitivity to inaccuracies of model and to interferences. A prospective approach to the problem is based on feedback control. Feedback control algorithms for passing through resonance zone of mechanical systems were considered in [6-8]. In [6] an optimal control algorithm for passage an unbalanced rotor through critical speed was proposed. For the same problem several control methods are evaluated, and the necessary number of dampers and their optimal location were determined in [7]. In [8] a nonlinear controller reducing resonance effects during despin of a dual-spin spacecraft was designed. A method of vibration suppression for rotating shafts passing through resonances by switching shafts stiffness was proposed in [9]. In [10] the dynamics of passage through resonance of a vibrating system with two degrees of freedom was examined. However, the early algorithms did not have enough robustness with respect to uncertainties and were hard to design.

For practical implementation of control system, it is important to develop reasonably simple passing through resonance zone control algorithms, which have such robustness property: keeping high quality of the controlled system (vibration unit) under variation of parameters and external conditions. Perhaps the first such a simple controller was proposed in [11] based on the speed-gradient method previously used for control of nonlinear oscillatory systems [12]. A number of speed-gradient algorithms for passage through resonance in 2-DOF systems were proposed in [13]. 
The approach of $[11,13]$ was applied to two-rotor vibration setup in [14].

This work is dedicated to further extension of the results of [14]. Problem statement for control of passage through resonance zone for mechanical systems with several degrees of freedom is adopted from [13]. The control algorithms based on the speed-gradient method for two-rotor vibration units with flexible cardan shafts are described. The simulation results illustrating efficiency and robustness of the proposed algorithms are presented. Previous results [14] are related to two-rotor vibration units with rigid cardan shafts.

\section{Problem Statement and Approach to Solution}

To describe the dynamics of a mechanical system and to carry out the control algorithm synthesis, it is easier to use standard Euler-Lagrange form, leading to the following model of controlled system dynamics with $\mathrm{n}$ degrees of freedom:

$$
A(q) \ddot{q}+C(q, \dot{q})+G(q)=B u,
$$

where $u=u(t)$ is $m$-dimensional input vector, representing elastic forces depending, in turn, on controlling forces; $q=$ $q(t)$ is $n$-vectors of generalized coordinates; $A(q)$ is $n \times$ $n$ - inertia matrix; $C(q, \dot{q})$ is the $n$-vector of Coriolis and centrifugal forces; $G(q)$ is the $n$-vector of gravity forces; $B$ is the $n \times m$ control matrix. For synthesis of control algorithms, it is often convenient to use equations in Hamiltonian form:

$$
\dot{q}=\left(\frac{\partial H}{\partial p}\right)^{T}, \quad \dot{p}=-\left(\frac{\partial H}{\partial q}\right)^{T}+B u,
$$

where $p=p(t)$ is the generalized momenta vector and $H=$ $H(p, q)$ denotes the Hamiltonian function (total energy of the system):

$$
H(p, q)=\frac{1}{2} p^{T}[A(q)]^{-1} p+\Pi(q)
$$

where $\Pi(q)$ is the potential energy. For design an idea of speed-gradient energy control is used and introduced in [12]. It is based on an auxiliary control goal: approach of free energy of the system to a surface of the given energy level:

$$
H(p(t), q(t)) \longrightarrow H^{*} \quad \text { when } t \longrightarrow \infty \text {. }
$$

Introducing the objective function

$$
Q(p, q)=\frac{1}{2}\left[H(p, q)-H^{*}\right]^{2},
$$

the goal (4) is reformulated as

$$
Q(q(t), p(t)) \longrightarrow 0 \quad \text { when } t \longrightarrow \infty
$$

Control algorithm for passing through resonant frequencies for an unbalanced rotor is based on the speed-gradient method [10], which allows us to synthesize control algorithms for significantly nonlinear objects. At the same time objective functional $Q(p, q)$ is chosen on the basis of total energy of a mechanical system excluding friction losses, because in this case total energy is invariant for a mechanical system that is required in the speed-gradient method in the version of [12].

It is assumed that in dynamics model of the system two subsystems are allocated: carrier and rotating body, such that the total energy is represented in the following form:

$$
\begin{aligned}
H & (p(t), q(t)) \\
& =H_{1}(p(t), q(t))+H_{2}(p(t), q(t))+H_{12}(p(t), q(t)),
\end{aligned}
$$

where $H_{1}$ is the energy of rotating subsystem, $H_{2}$ is the energy of a carrier subsystem, and $H_{12}$ is the energy of interaction.

The solution of $[6,8]$ adopted in this paper is based upon usage of the speed gradient algorithms [12] and a motion separation into fast and slow components, which occurs near resonance zone [15]. Quantitative analysis of slow "pendularlike" movements $\Psi(t)$ was performed by Blekhman et al. in [15] where the frequency of an "internal pendulum" was evaluated. It will be further called the "Blekhman frequency." An approach of [15] is briefly described below for completeness.

In [15] the following system with inertial excitation of oscillations was considered:

$$
\begin{gathered}
I \ddot{\varphi}=L(\dot{\varphi})-R(\dot{\varphi})+m \varepsilon \ddot{x} \sin \varphi, \\
m_{1} \ddot{x}+\beta \dot{x}+c x=m \varepsilon\left(\dot{\varphi}^{2} \cos \varphi+\ddot{\varphi} \sin \varphi\right),
\end{gathered}
$$

where $\varphi$ is the rotation angle of a rotor, $x$ is a platform deflection, $M$ is the mass of a platform, $m$ is the rotor mass, $I$ is the inertia moment of a rotor, $m_{1}=M+m$ is the system mass, $c$ is the coefficient of axial stiffness, $\beta$ is the damping coefficient of the spring, $\varepsilon$ is the eccentricity of rotors, $L(\dot{\varphi})$ is the rotation torque of a motor (static characteristics), and $R(\dot{\varphi})$ is the torque of resistance forces. Assuming that frequency of rotation $\omega$ varies slowly in terms of

$$
\frac{\dot{\omega}}{\omega} \ll \omega
$$

and using the method of direct separation of motions [1], Blekhman et al. derived the equation of slow "pendular-like" oscillations in the following form [15]:

$$
\ddot{\Psi}+2 n_{1} \dot{\Psi}+b \sin \Psi-\rho^{2} \sin ^{2} \frac{\Psi}{2}=0,
$$

where $\Psi$ is slow variable: "addition" to rotation frequency, $2 n_{1}=k / I, k=k_{L}+k_{R}\left(k_{L}>0, k_{R}>0\right)$ is the "total damping coefficient," obtained by linearization of expressions for $L(\dot{\varphi})$ and $R(\dot{\varphi})$ near value $\dot{\varphi}=\omega$ :

$$
\begin{gathered}
L(\omega+\dot{\Psi})=L(\omega)-k_{L} \dot{\Psi}, \quad R(\omega+\dot{\Psi})=R(\omega)-k_{R} \dot{\Psi}, \\
b=\frac{\left(m \varepsilon \omega^{2}\right)^{2}}{2 m_{1} I} \frac{\omega^{* 2}-\omega^{2}}{\left(\omega^{* 2}-\omega^{2}\right)^{2}+4 n^{2} \omega^{2}}=\frac{1}{2} \frac{m_{1}}{I}\left(\omega^{* 2}-\omega^{2}\right) A^{2} ; \\
\rho^{2}=\frac{\left(m \varepsilon \omega^{2}\right)^{2}}{2 m_{1} I} \frac{2 n \omega}{\left(\omega^{* 2}-\omega^{2}\right)^{2}+4 n^{2} \omega^{2}}=\frac{m_{1}}{I} 2 n \omega A^{2} ;
\end{gathered}
$$




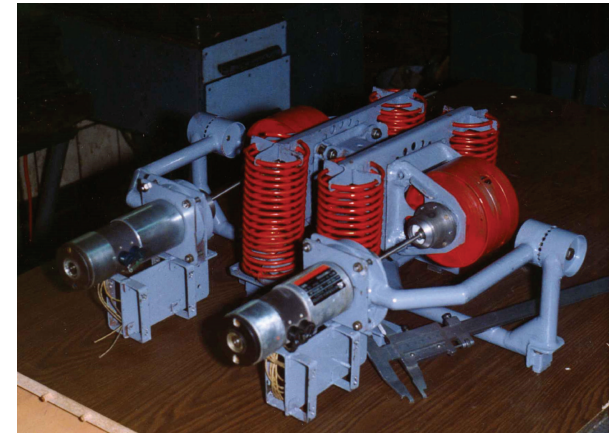

(a)

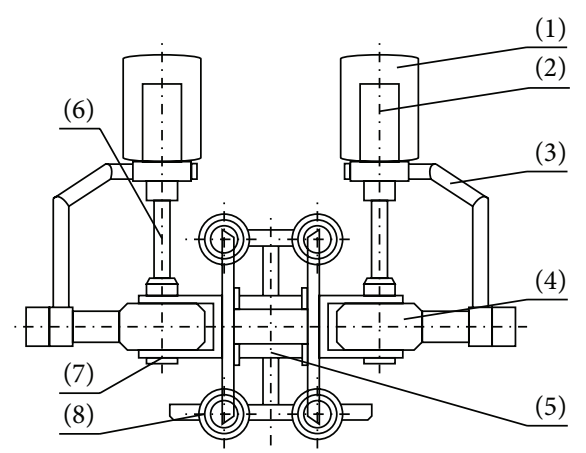

(b)

FiguRE 1: Two-rotor vibration unit with elastic cardan shafts: (a) general view and (b) schematics-top view ((1) motors, (2) motor supports, (3) frame of the unit, (4) unbalanced rotors, (5) vibrating platform, (6) cardan shafts, (7) rotor bearings, and (8) springs).

$\omega^{* 2}=c / m_{1}$ is a resonance frequency, $2 n=\beta / m_{1}$, and $A=$ $m \varepsilon \omega^{2} / M \sqrt{\left(\omega^{* 2}-\omega^{2}\right)^{2}+4 n^{2} \omega^{2}}$ is the first approximation to amplitude of oscillations of a platform. If the relation $\dot{\omega} / \omega \ll$ $\omega$ holds in the preresonance zone $\left(\omega<\omega^{*}\right)$ then the value

$$
\omega_{B}=\sqrt{b}=A \sqrt{\frac{m_{1}\left(\omega^{* 2}-\omega^{2}\right)}{2 I}}
$$

appears to be a frequency of small free oscillations of an "internal pendulum" (excluding resistance force), which is called the "Blekhman frequency" thereafter.

As seen before, that frequency vanishes to zero when $\omega=\omega^{*}$. The necessary condition of the validity of (10) is that the Blekhman frequency $\omega_{B}$ should be significantly less than frequency of rotations $\omega$ (usually, $\omega_{B} / \omega<1 / 3$ is typically sufficient).

The idea of control algorithms described below is to extract the slow motion $\Psi(t)$ and to swing it with the aim to increase the energy of the rotating subsystem. To isolate slow motions, low-pass filter is inserted into the energy control algorithms. Particularly, if slow component appears in oscillations of angular velocity of a rotor $\dot{\varphi}$, then the control algorithm proposed in [11] is used:

$$
u=-\gamma \operatorname{sign}\left(\left(H-H^{*}\right) \dot{\psi}\right), \quad T_{\psi} \dot{\psi}=-\psi+\dot{\varphi},
$$

where $H=H(p, q)$ denotes the Hamiltonian (total energy of the system), $\psi(t)$ is the variable of a filter that is an estimate of the slow motions $\Psi(t)$ satisfying (10), and $T_{\psi}$ is the time constant of a filter. At low damping, slow motions also fade out slowly, which gives control algorithm an opportunity to create suitable conditions to pass through resonance zone. Thus, the effect of "feedback resonance" [16] is created. After passing the resonance zone it is suggested to turn off the "swinging" and then to switch off control and to apply the constant drive torque. For a proper work of a filter, it should suppress fast oscillations with frequency $\omega$ and pass slow oscillations with $\omega_{B}$ frequency, where $\omega_{B}$ is the Blekhman frequency. That is, time constant of a filter $T_{\psi}$ should be chosen from the inequality

$$
T_{\psi}<\frac{2 \pi}{\omega_{B}}
$$

Algorithms of passing through resonance zone for the tworotor vibration units are described in [14]. Below we analyze the algorithm of [14] taking into account elasticity of the cardan shafts.

\section{Passing through Resonance Control Algorithm of Two-Rotor Vibration Unit}

Consider the two-rotor vibration unit in the startup/spin-up mode [17]. The unit consists of two rotors 4 installed on the vibrating platform 5 . The rotors are elastically connected with fixed basis 2 , and frame 3 by springs 8 (Figure 1).

Since the motors are located on motionless supports and the unbalanced rotors are mounted on the vibrating platform (bearings), the links implemented via cardan shafts should be considered as elastic ones (Figure 2). In this paper, only torsional elasticity will be taken into account.

The block scheme representing the elastic links of drive shafts with unbalanced rotors may be presented in the following form (Figure 3), where $M_{i}, i=1,2$ are the electromechanical torques of motors; $\omega_{i}$ are the angular velocities of motors; $M_{c i}=k_{c} \cdot \dot{\varphi}_{i}$ are resistance moments of the rotors mounted on the vibrating platform; $M_{r i}$ are the unbalanced rotor's own moments.

$M_{r i}(t)=m \rho g \cos \left(\varphi+\varphi_{i}\right) ; \widetilde{M}_{\mathrm{pi}}$ are the moments, caused by the platform influence:

$$
\begin{aligned}
\widetilde{M}_{\mathrm{pi}}= & -\ddot{x}_{c} m \rho \sin \left(\varphi+\varphi_{i}\right)+\ddot{y}_{c} m \rho \cos \left(\varphi+\varphi_{i}\right) \\
& +\ddot{\varphi}\left(J_{i}+(-1)^{i} r m \rho \cos \varphi_{i}\right)+(-1)^{i} \dot{\varphi}^{2} r m \rho \sin \varphi_{i} ;
\end{aligned}
$$

$c_{B}, b_{B}$ are the stiffness and damping coefficients of the cardan shafts, $\varphi, \varphi_{1}$, and $\varphi_{2}$ are angle of the support and rotation angles of the rotors, respectively, measured from the horizontal position, $x_{c}, y_{c}$ are the horizontal and vertical displacements of the vibrating platform center $O^{\prime}$ from its equilibrium position $O, m_{i}=m, i=1,2$, and $m_{n}$ are the masses of the rotors and supporting body, $J_{1}=J_{2}=J$ are the inertia moments of the rotors, $\rho_{i}=\rho, i=1,2$ are the rotor eccentricities, $c_{01}, c_{02}$ are the horizontal and vertical spring stiffness, $g$ is the gravity acceleration, $m_{0}$ is the total mass of 


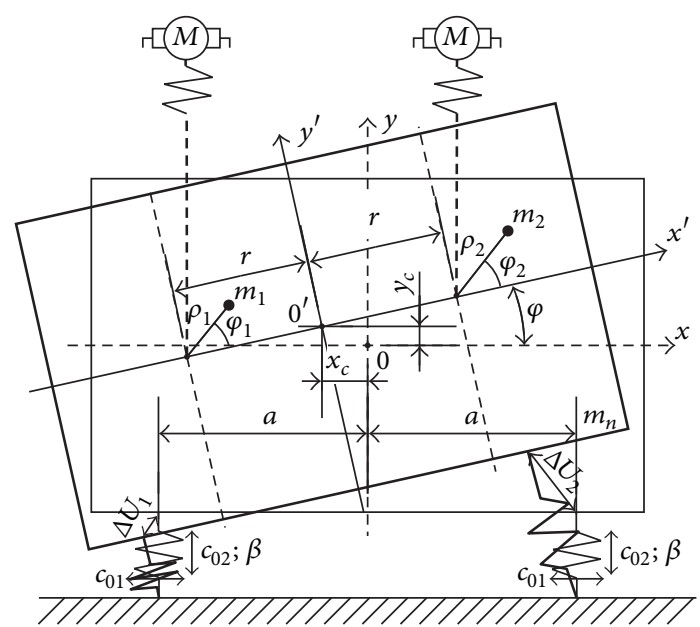

FIGURE 2: Schematics of the two-rotor vibration unit with elastic cardan shafts.

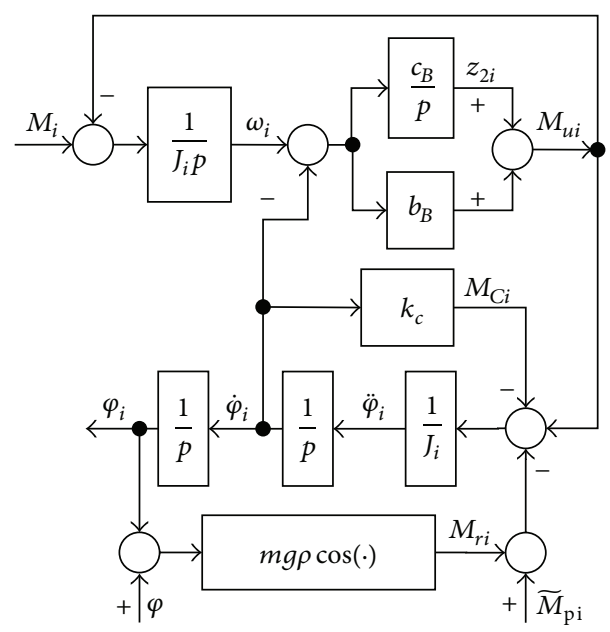

FIGURE 3: Block scheme, representing the elastic links of the motors with unbalanced rotors.

the unit, $m_{0}=2 m+m_{n}, \beta$ is the damping coefficient, $k_{c}$ is the friction coefficient in the bearings, $M_{i}=u_{i}(t)$ are the motor torques (controlling variables), and $r$ is the distance from the centers of the rotors to $O^{\prime}$. It is assumed that the rotors are identical and the rotor shafts are orthogonal to the motion of the support.

To convert the scheme into the state space equations, accounting elasticity, let us substitute the state variables $z_{1 i}=$ $\omega_{i}$ and $z_{2 i}$ as the outputs of corresponding integrators $(i=$ $1,2)$. Then the dynamics of the cardan shafts taking into account torsional elasticity are described by the differential equations:

$$
\begin{aligned}
& \dot{z}_{1 i}=\frac{1}{J_{i}} \cdot M_{i}-\frac{1}{J_{i}} \cdot M_{u i}=\frac{1}{J_{i}} \cdot M_{i}-\frac{1}{J_{i}} \cdot\left(z_{2 i}+b_{B}\left(z_{1 i}-\dot{\varphi}_{i}\right)\right), \\
& \dot{z}_{2 i}=c_{B}\left(z_{1 i}-\dot{\varphi}_{i}\right), \quad M_{u i}=z_{2 i}+b_{B}\left(z_{1 i}-\dot{\varphi}\right) .
\end{aligned}
$$

Assume that the whole system dynamics may be considered in the vertical plane. Then the equations of dynamics have the following form [8]:

$$
\begin{aligned}
& m_{0} \ddot{x}_{c}-\ddot{\varphi} m \rho\left[\sin \left(\varphi+\varphi_{1}\right)+\sin \left(\varphi+\varphi_{2}\right)\right] \\
& -\ddot{\varphi}_{1} m \rho \sin \left(\varphi+\varphi_{1}\right)-\ddot{\varphi}_{2} m \rho \sin \left(\varphi+\varphi_{2}\right) \\
& -\dot{\varphi}^{2} m \rho\left[\cos \left(\varphi+\varphi_{1}\right)+\cos \left(\varphi+\varphi_{2}\right)\right] \\
& -\dot{\varphi}_{1}^{2} m \rho \cos \left(\varphi+\varphi_{1}\right)-\dot{\varphi}_{2}^{2} m \rho \cos \left(\varphi+\varphi_{2}\right) \\
& -2 \dot{\varphi} \dot{\varphi}_{1} m \rho \cos \left(\varphi+\varphi_{1}\right)-2 \dot{\varphi} \dot{\varphi}_{2} m \rho \cos \left(\varphi+\varphi_{2}\right) \\
& +2 c_{01} x_{c}+\beta \dot{x}_{c}=0 \\
& m_{0} \ddot{y}_{c}+\ddot{\varphi} m \rho\left[\cos \left(\varphi+\varphi_{1}\right)+\cos \left(\varphi+\varphi_{2}\right)\right] \\
& -\ddot{\varphi}_{1} m \rho \cos \left(\varphi+\varphi_{1}\right)-\ddot{\varphi}_{2} m \rho \cos \left(\varphi+\varphi_{2}\right) \\
& -\dot{\varphi}^{2} m \rho\left[\sin \left(\varphi+\varphi_{1}\right)+\sin \left(\varphi+\varphi_{2}\right)\right] \\
& -\dot{\varphi}_{1}^{2} m \rho \sin \left(\varphi+\varphi_{1}\right)-\dot{\varphi}_{2}^{2} m \rho \sin \left(\varphi+\varphi_{2}\right) \\
& -2 \dot{\varphi} \dot{\varphi}_{1} m \rho \sin \left(\varphi+\varphi_{1}\right)-2 \dot{\varphi} \dot{\varphi}_{2} m \rho \sin \left(\varphi+\varphi_{2}\right) \\
& +m_{0} g+2 c_{02} y_{c}+\beta \dot{y}_{c}=0 ; \\
& -\ddot{x}_{c} m \rho\left[\sin \left(\varphi+\varphi_{1}\right)+\sin \left(\varphi+\varphi_{2}\right)\right] \\
& +\ddot{y}_{c} m \rho\left[\cos \left(\varphi+\varphi_{1}\right)+\cos \left(\varphi+\varphi_{2}\right)\right] \\
& +\ddot{\varphi}\left[J+J_{1}+J_{2}-2 r m \rho\left(\cos \varphi_{1}-\cos \varphi_{2}\right)\right] \\
& +\ddot{\varphi}_{1}\left(J_{1}-r m \rho \cos \varphi_{1}\right)+\ddot{\varphi}_{2}\left(J_{2}+r m \rho \cos \varphi_{2}\right) \\
& +\dot{\varphi}_{1}^{2} r m \rho \sin \varphi_{1}-\dot{\varphi}_{2}^{2} r m \rho \sin \varphi_{2}+2 r m \rho \dot{\varphi} \dot{\varphi}_{1} \sin \varphi_{1} \\
& -2 r m \rho \dot{\varphi} \dot{\varphi}_{2} \sin \varphi_{2}+m \rho g\left[\cos \left(\varphi+\varphi_{1}\right)+\cos \left(\varphi+\varphi_{2}\right)\right] \\
& +c_{03} \varphi+\beta \dot{\varphi}=0, \\
& -\ddot{x}_{c} m \rho \sin \left(\varphi+\varphi_{1}\right)+\ddot{y}_{c} m \rho \cos \left(\varphi+\varphi_{1}\right) \\
& +\ddot{\varphi}\left(J_{1}-r m \rho \cos \varphi_{1}\right)+\ddot{\varphi}_{1} J_{1}-\dot{\varphi}^{2} r m \rho \sin \varphi_{1} \\
& +m \rho g \cos \left(\varphi+\varphi_{1}\right)+k_{c} \dot{\varphi}_{1}=M_{u 1} ; \\
& -\ddot{x}_{c} m \rho \sin \left(\varphi+\varphi_{2}\right)+\ddot{y}_{c} m \rho \cos \left(\varphi+\varphi_{2}\right) \\
& +\ddot{\varphi}\left(J_{2}+r m \rho \cos \varphi_{1}\right)+\ddot{\varphi}_{2} J_{2}+\dot{\varphi}^{2} r m \rho \sin \varphi_{2} \\
& +m \rho g \cos \left(\varphi+\varphi_{2}\right)+k_{c} \dot{\varphi}_{2}=M_{u 2} .
\end{aligned}
$$

At the low levels of constant control action $u_{i}(t) \equiv(-1)^{i} M_{0}$, $i=1,2$ in the near-resonance zone the rotor angle is "captured," while increase of the control torque leads to passage through resonance zone towards the desired angular velocity. The simulation results for system (16), (17) are shown in Figure 4 with basic system parameters: $J_{i}=0.014 \mathrm{~kg} \cdot \mathrm{m}^{2}$, $m=1.5 \mathrm{~kg}, m_{n}=9 \mathrm{~kg}, \rho=0.04 \mathrm{~m}, k_{c}=0.01 \mathrm{~J} / \mathrm{s}, \beta=5 \mathrm{~kg} / \mathrm{s}$, $c_{02}=5300 \mathrm{~N} / \mathrm{m}, c_{01}=1300 \mathrm{~N} / \mathrm{m}$, and constant torque $M_{0}=$ $0.65 \mathrm{~N} \cdot \mathrm{m}$ (internal curves, "capture") and $M_{0}=0.66 \mathrm{~N} \cdot \mathrm{m}$ 


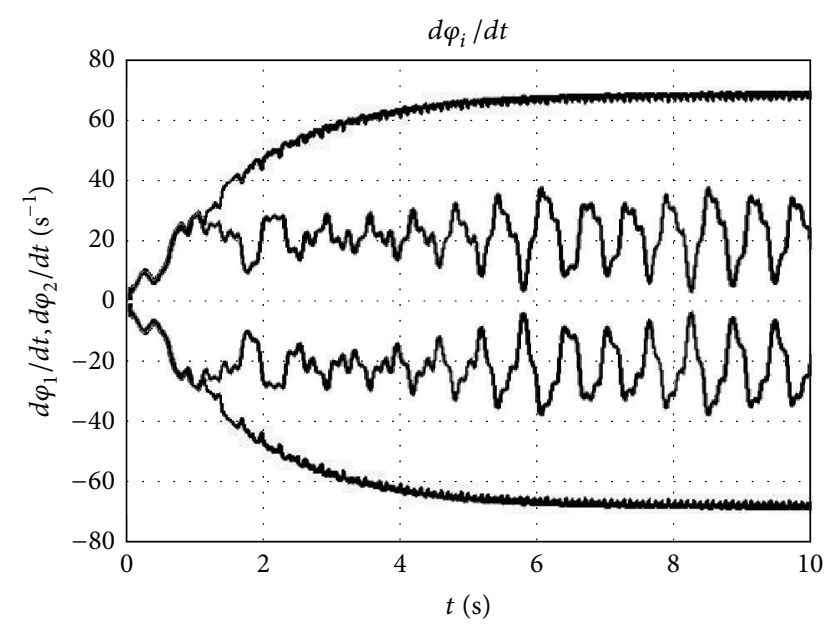

FIgURE 4: Constant drive torque, $u_{i}(t) \equiv(-1)^{i} M_{0}, M_{0}=0.65 \mathrm{~N} \cdot \mathrm{m}$ (internal curves, "capture"), and $M_{0}=0.66 \mathrm{~N} \cdot \mathrm{m}$ (external curves, passing).

(external curves, passage). Simulation of system (17) without elasticity $\left(M_{u i}=M_{i}\right)$ and without control $\left(M_{i}=u_{i}=\right.$ $\left.(-1)^{i} M_{0}\right)$ has shown that the lowest constant drive torque $M_{0}$ providing passing through the resonance is $0.66 \mathrm{~N} \cdot \mathrm{m}$ (Figure 4).

Like in a number of previous papers the feedback control algorithm $u=\mathbf{U}(z)$ where

$$
z=\left[x_{c}, \dot{x}_{c}, y_{c}, \dot{y}_{c}, \varphi, \dot{\varphi}, \varphi_{1}, \dot{\varphi}_{1}, \varphi_{2}, \dot{\varphi}_{2}\right]^{T}
$$

is the state vector of the system that is used. The control goal is passing through resonance zone to achieve the desired level of the velocity of the unbalanced rotors. It is assumed that the level of control is limited and does not allow system to pass through the resonance zone using constant driving torque.

As seen in Figure 4 the rotational motions separate into fast and slow ones, that is, the proposed approach applies. The following modification of control Algorithm (13) proposed in [14] for passing through resonance zone was studied in this paper:

$$
\begin{aligned}
& u_{i}= \begin{cases}(-1)^{i} M_{0}, & \text { if } \gamma=1, \\
(-1)^{i} M_{0}, & \text { if } \gamma=0,\left(H-H^{*}\right)\left(\dot{\varphi}_{i}-\psi_{i}\right)>0, \\
0, & \text { else, }\end{cases} \\
& T_{\psi} \dot{\psi}_{i}=-\psi_{i}+\dot{\varphi}_{i}, \quad i=1,2,
\end{aligned}
$$

where $H=T+\Pi, \psi_{i}(t)$ are the variables of the filters, $T_{\psi}>$ $0, T_{\psi}=$ const, and $H^{*}$ are the parameters of the algorithm, and $\gamma(t)=\max _{0 \leq \tau<t} \operatorname{sgn}\left(H(\tau)-H^{*}\right)$, where sgn $[z]=1$ with $z>0, \operatorname{sgn}[z]=0$ with $z \leq 0$. Time constant of the filters $T_{\psi}$ should satisfy relation (14).

Efficiency of control Algorithm (19) was studied in the MATLAB environment. The relative simulation error does not exceed 5\%. Calculations were made with the same values of the basic parameters of the system. Simulation of system (17) without elasticity $\left(M_{u i}=M_{i}\right)$ and with control

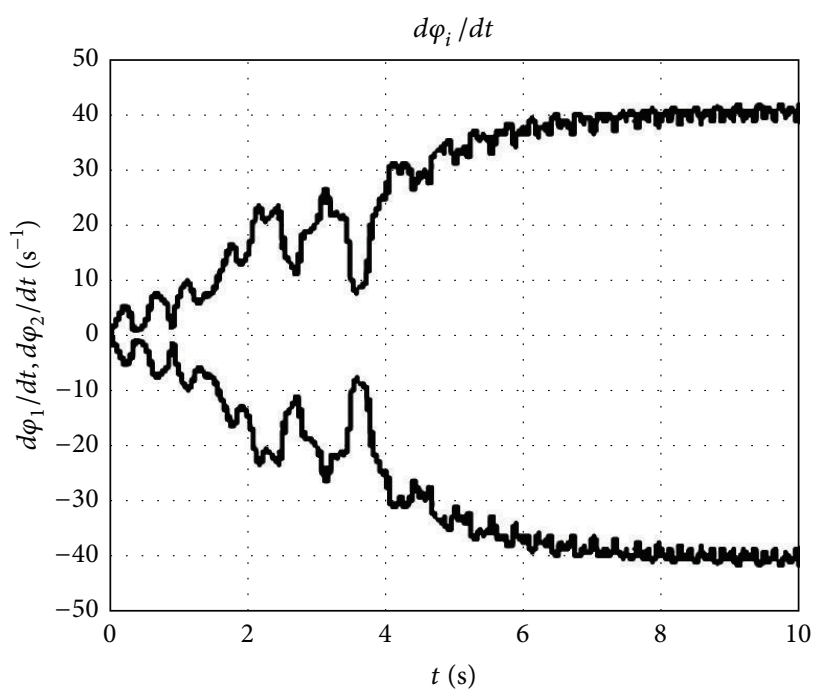

FIGURE 5: Simulation results for system (17), (19) with $M_{0}=$ $0.45 \mathrm{~N} \cdot \mathrm{m}$.
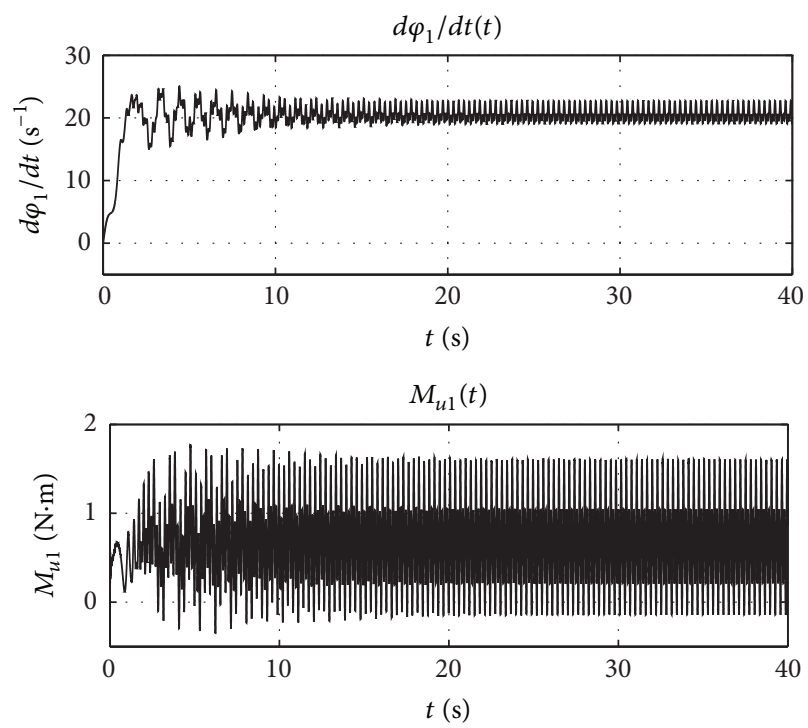

FIGURE 6: Simulation results for the system with elasticity (16), (17) and with constant drive torque $M_{0}=0.66 \mathrm{~N} \cdot \mathrm{m}$.

Algorithm (19) has shown that passage through the resonance zone occurs for $M_{0} \geq 0.36 \mathrm{~N} \cdot \mathrm{m}$. Figure 5 demonstrates the graphs of $\dot{\varphi}_{1}$ and $\dot{\varphi}_{2}$ for system with Algorithm (19) for operating value of the torque $M_{0}=0.45 \mathrm{~N} \cdot \mathrm{m}$ without elasticity.

Simulation results for the system with elasticity (16), (17) and without control are shown in Figure 6 for $M_{0}=$ $0.66 \mathrm{~N} \cdot \mathrm{m}$. It is seen that the rotor angular velocity achieves the steady state near resonance: $\omega_{\text {res }}=21.5 \mathrm{~s}^{-1}$, while elastic torque $M_{u 1}(t)$ exhibits persisting oscillations with the amplitude exceeding the threshold value for rigid system $M_{0}=0.66 \mathrm{~N} \cdot \mathrm{m}$. It means that passing through resonance for elastic system requires bigger drive torque. 


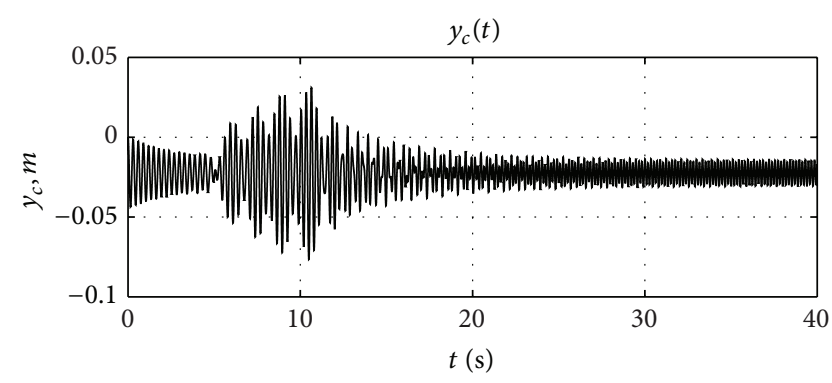

FIGURE 7: $y_{c}(t)$ : vertical motion of the platform for $M_{0}=0.36 \mathrm{~N} \cdot \mathrm{m}$.

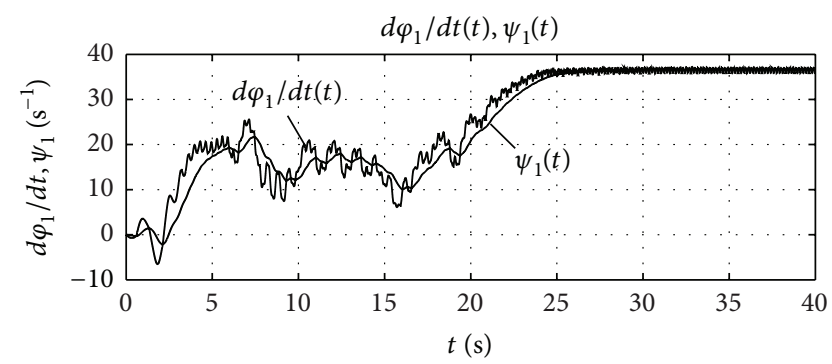

FIGURE 8: $\dot{\varphi}_{1}(t)$ : rotor angular velocity; $\psi_{1}$ : value of the filter variable for $M_{0}=0.36 \mathrm{~N} \cdot \mathrm{m}$.

TABLE 1

\begin{tabular}{lcc}
\hline$c_{B}$ & $t_{\text {res }}, \mathrm{s}$ & $t_{\mathrm{tr}}, \mathrm{s}$ \\
\hline 3000 & 19.4 & 24.5 \\
2500 & 19.42 & 24.05 \\
2000 & 18.2 & 22.0 \\
1500 & 18.7 & 23.0 \\
1000 & 19.4 & 24.03 \\
\hline
\end{tabular}

Quantitative characteristics of dynamics of the unit with elasticity (16), (17) with control Algorithm (19) for $M_{0}=$ $0.36 \mathrm{~N} \cdot \mathrm{m}, c_{B}=3000(\mathrm{~N} \cdot \mathrm{m} / \mathrm{rad}), b_{B}=0.3(\mathrm{~N} \cdot \mathrm{m} \cdot \mathrm{s})$, and $T_{\psi}=1.0 \mathrm{~s}$ are presented in Figures $7-10$. The above parameter values correspond to the frequency of torsion oscillations of the shafts $\omega \approx 750 \mathrm{~s}^{-1}$.

It is seen from the pictures in Figures 7-10 that time for passing through resonance is $t_{\text {res }}=19.4 \mathrm{~s}$, and time of approaching to the desired velocity is $t_{\mathrm{tr}}=24.5 \mathrm{~s}$. It is also seen that after passing the resonance zone the platform oscillates with steady state amplitude $A=0.8 \mathrm{~cm}$, while elastic torque $M_{u 1}$ at the near-resonance zone reaches the value of $M_{u 1}=1.2 \mathrm{~N} \cdot \mathrm{m}$. It settles in a range $M_{u 1} \leq 0.85 \mathrm{~N} \cdot \mathrm{m}$, and the difference between motor velocity and rotor velocity settles at the level of $\left|\dot{\varphi}_{1}-\omega_{1}\right| \leq 0.1 \mathrm{~s}^{-1}$, that is, $0.3 \%$ from the steady velocity of the rotor.

In Figures 11 and 12 the plots of $\dot{\varphi}_{1}$ for system (17), (19) (without elasticity, Figure 11) and for system (16), (17), (19) (taking into account the shaft elasticity, Figure 12) are presented. The value of parameter $M_{0}$ in Algorithm (19) is different: $M_{0}=0.4 \mathrm{~N} \cdot \mathrm{m}$. As seen from the picture, passing through resonance in the first case takes longer time.

The study of the algorithm efficiency for different values of the elasticity parameters is presented in Tables 1 and 2 .

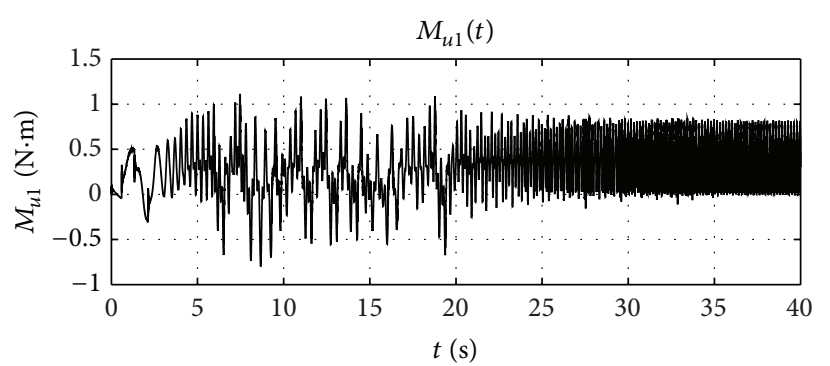

FIGURE 9: $M_{u 1}(t)$ : elastic torque for $M_{0}=0.36 \mathrm{~N} \cdot \mathrm{m}$.

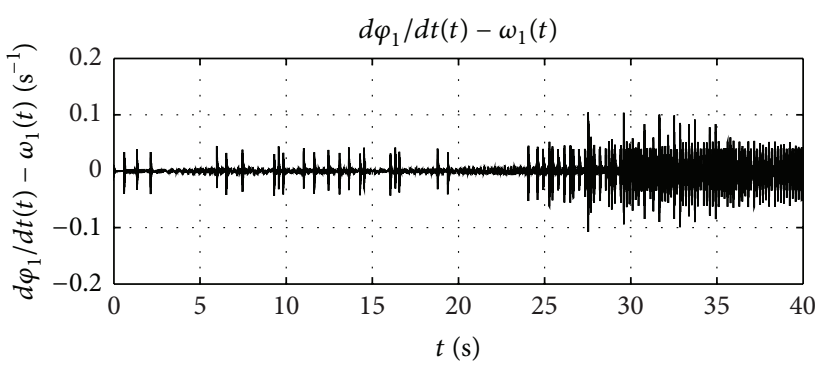

FIGURE 10: $\dot{\varphi}_{1}(t)-\omega_{1}(t)$ difference between velocity of an unbalanced rotor and motor velocity for $M_{0}=0.36 \mathrm{~N} \cdot \mathrm{m}$.

TABLE 2

\begin{tabular}{lcc}
\hline$b_{B}$ & $t_{\text {res }}, \mathrm{s}$ & $t_{\mathrm{tr}}, \mathrm{s}$ \\
\hline 0.8 & 19.42 & 24.0 \\
0.7 & 19.01 & 23.5 \\
2000 & 19.42 & 24.0 \\
1500 & 13.06 & 17.98 \\
1000 & 19.4 & 24.5 \\
\hline
\end{tabular}

In Table 1 the times of passing through resonance $t_{\text {res }}$, s and times of achieving the steady state mode $t_{\mathrm{tr}}$, $s$ for different stiffness coefficients $c_{B}$ are presented. In Table 2 the values of the coefficient $b_{B}$ for fixed $c_{B}=3000$ are given.

As seen from the presented graphs, control algorithm for passing through resonance synthesized without accounting of drive shaft elasticity is efficient if the drive shaft elasticity is taken into account as well. Moreover, as the simulation shows, with the same algorithm parameters, varying the values of the elastic connection can increase the passing through resonance time constant by 1.5-2 times and the time of entering the given velocity is increased by $40 \%$, but control objective is completed in all cases. The computer simulation also shows that using the passing through resonance control algorithm the level of control torque can be reduced by over 55\%, in comparison to traditional control with constant torque.

Thus, using control Algorithm (19), the level of control action required for passing through resonance can be significantly reduced. 


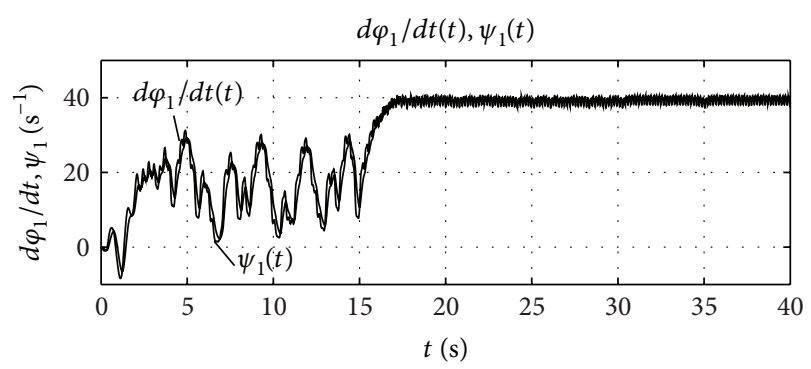

Figure 11: Plots of $\dot{\varphi}_{1}(t) \psi_{1}$ for $M_{0}=0.4 \mathrm{~N} \cdot \mathrm{m}$. Elasticity is not taken into account.

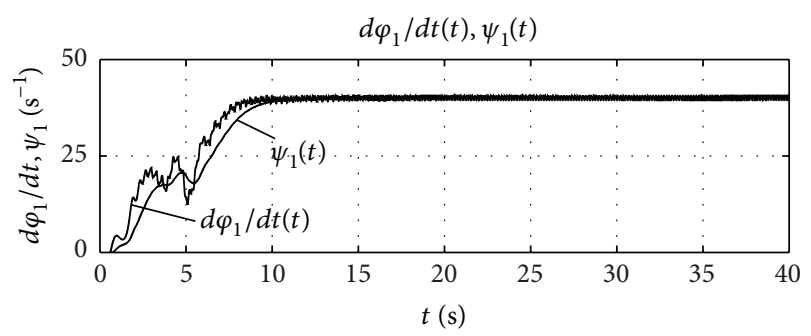

FIGURE 12: Plots of $\dot{\varphi}_{1}(t) \psi_{1}$ for $M_{0}=0.4 \mathrm{~N} \cdot \mathrm{m}$. Elasticity is taken into account.

\section{Conclusion}

In this paper the algorithm for controlled passage through resonance zone proposed in $[13,14]$ is analyzed taking into account elasticity of the cardan shafts. The simulation results show that the approach for design of simple control algorithms for passing through resonance zone in vibration units based on "swinging" of slow oscillations of an angular velocity of the rotors, proposed in $[11,13]$ applies to vibration units with elasticity. For constant drive torques passage through resonance requires bigger value of the motor torque, while for controlled torque passage through resonance is achieved with the same value of the motor torque, even for elastic shafts. Even more, for units with flexible cardan shafts it may become more efficient than for rigid units. A possible reason is that additional vibrations caused by elasticity increase amplitude value of rotor angular velocity that may help in passing through resonance.

The design of control algorithm is simple since it has only two tunable parameters. In addition, the value of the parameter $H^{*}$ (desired energy of the system) is not necessarily related to the working regime of the vibration unit. The value of $H^{*}$ should meet the only requirement that it should exceed maximum energy of the system in the resonance zone. To achieve the working regime the only parameter to be chosen is the gain $\gamma$.

Next step of the research is examination of the closed loop system performance for an ensemble of initial conditions, including asymmetrical ones, like it was done for rigid system [14].

\section{Conflict of Interests}

The authors declare that there is no conflict of interests regarding the publication of this paper.

\section{Acknowledgments}

The authors are grateful to Dmitry Gorlatov for helping with simulation and formatting of the paper. The work was performed in IPME RAS and supported by Russian Scientific Foundation (Project 14-29-00142).

\section{References}

[1] I. I. Blekhman, Vibrational Mechanics, World Scientific, Singapore, 2000.

[2] V. O. Kononenko, Oscillating Systems with Bounded Excitation, Nauka, Moscow, Russia, 1964, (Russian).

[3] D. Quinn, R. Rand, and J. Bridge, "The dynamics of resonant capture," Nonlinear Dynamics, vol. 8, no. 1, pp. 1-20, 1995.

[4] L. Cvetićanin, "Dynamics of the non-ideal mechanical systems: a review," Journal of the Serbian Society for Computational Mechanics, vol. 4, no. 2, pp. 75-86, 2010.

[5] V. V. Gortinskii, A. D. Savin, A. B. Demskii, M. A. Boriskin, and E. A. Alabin, "A technique for reducing resonance amplitudes during start of vibration machines," Patent of USSR no. 255760, 28.10.1969, Bulletin no. 33, 1969 (Russian).

[6] L. N. Malinin and A. A. Pervozvansky, "Optimization of passage an unbalanced rotor through critical speed," Mashinovedenie, no. 4, pp. 36-41, 1983 (Russian).

[7] Z. Viderman and I. Porat, "An optimal control method for passage of a flexible rotor through resonances," Journal of Dynamic Systems, Measurement, and Control, vol. 109, no. 3, pp. 216-223, 1987.

[8] R. J. Kinsey, D. L. Mingori, and R. H. Rand, "Nonlinear controller to reduce resonance effects during despin of a dualspin spacecraft through precession phase lock," in Proceedings of the 31st IEEE Conference on Decision and Control, vol. 4, pp. 3025-3030, Tucson, Ariz, USA, December 1992.

[9] J. Wauer and S. Suherman, "Vibration suppression of rotating shafts passing through resonances by switching shaft stiffness," Journal of Vibration and Acoustics-Transactions of the ASME, vol. 120, no. 1, pp. 170-180, 1998.

[10] J. M. Balthazar, B. I. Cheshankov, D. T. Ruschev, L. Barbanti, and H. I. Weber, "Remarks on the passage through resonance of a vibrating system with two degrees of freedom, excited by a non-ideal energy source," Journal of Sound and Vibration, vol. 239, no. 5, pp. 1075-1085, 2001.

[11] O. P. Tomchina, "Passing through resonances in vibratory actuators by speed-gradient control and averaging," in Proceedings of the International Conference on Control of Oscillations and Chaos (COC '97), vol. 1, pp. 138-141, IEEE, Saint Petersburg, Russia, August 1997.

[12] B. R. Andrievskii, P. Y. Guzenko, and A. L. Fradkov, "Control of nonlinear vibrations of mechanical systems via the speed gradient method," Automation and Remote Control, vol. 57, no. 4, pp. 456-467, 1996.

[13] A. Fradkov, O. Tomchina, and D. Tomchin, "Controlled passage through resonance in mechanical systems," Journal of Sound and Vibration, vol. 330, no. 6, pp. 1065-1073, 2011. 
[14] A. L. Fradkov, D. A. Tomchin, and O. P. Tomchina, "Controlled passage through resonance for two-rotor vibration unit," in Mechanics and Model-Based Control of Advanced Engineering Systems, A. K. Belyaev, H. Irschik, and M. Krommer, Eds., pp. 95-102, Springer, Wien, Vienna, 2014.

[15] I. I. Blekhman, D. A. Indeitsev, and A. L. Fradkov, "Slow motions in systems with inertial excitation of vibrations," Journal of Machinery Manufacture and Reliability, vol. 37, no. 1, pp. 21-27, 2008.

[16] A. Fradkov, "Exploring nonlinearity by feedback," Physica D: Nonlinear Phenomena, vol. 128, no. 2-4, pp. 159-168, 1999.

[17] I. I. Blekhman, Y. A. Bortsov, A. A. Burmistrov et al., "Computer-controlled vibrational set-up for education and research," in Proceedings of the 14th IFAC World Congress, vol. M, pp. 193-197, 1999. 


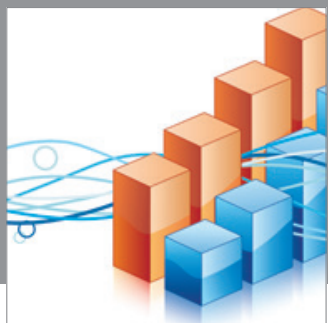

Advances in

Operations Research

mansans

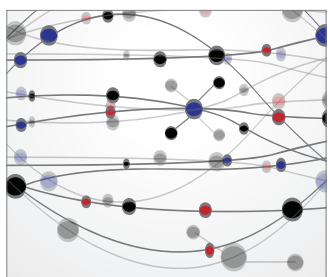

The Scientific World Journal
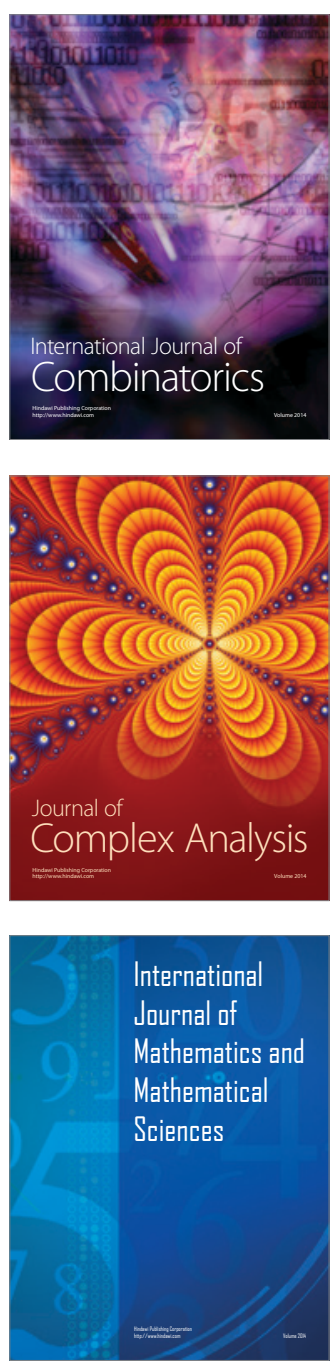
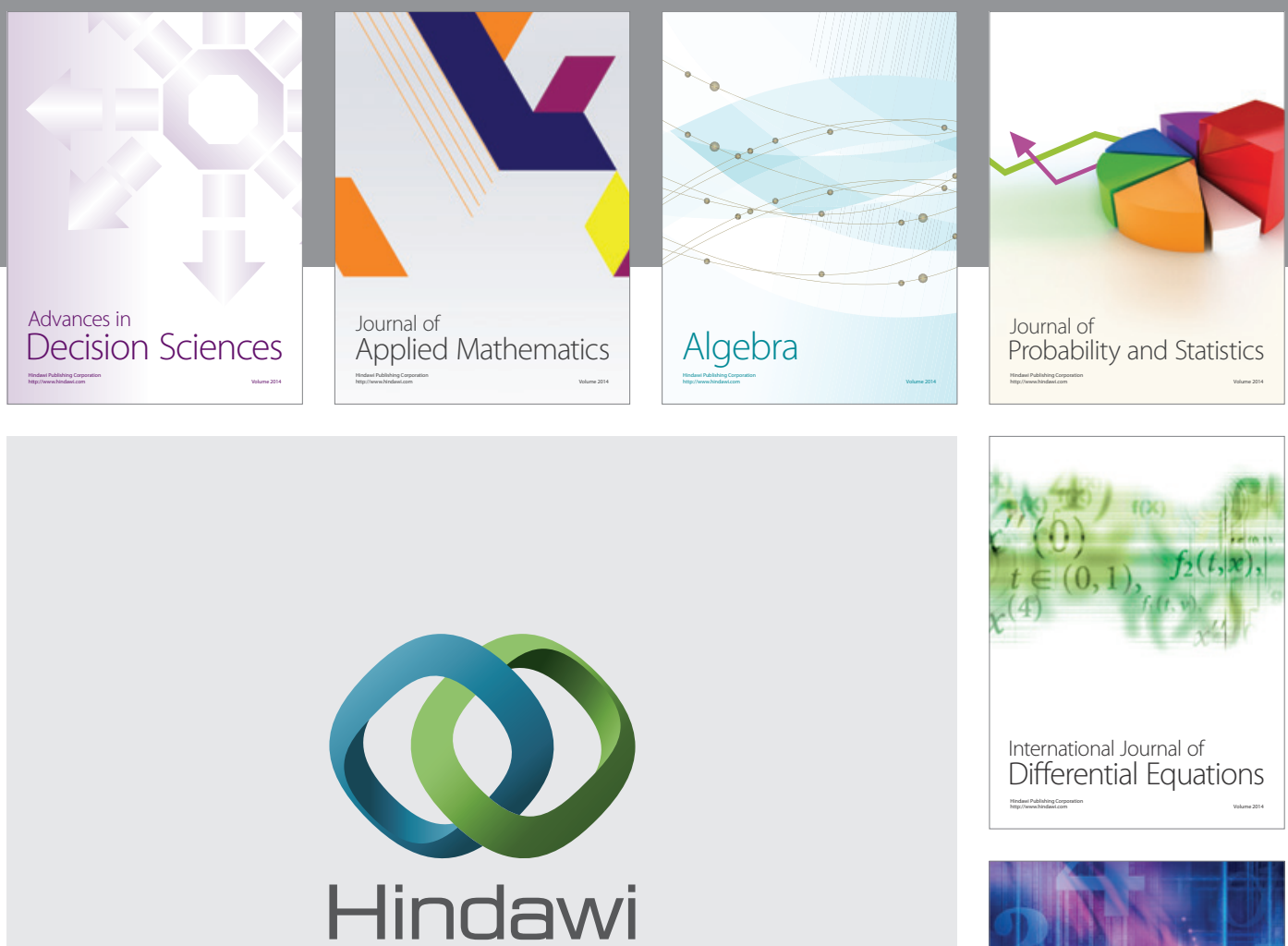

Submit your manuscripts at http://www.hindawi.com
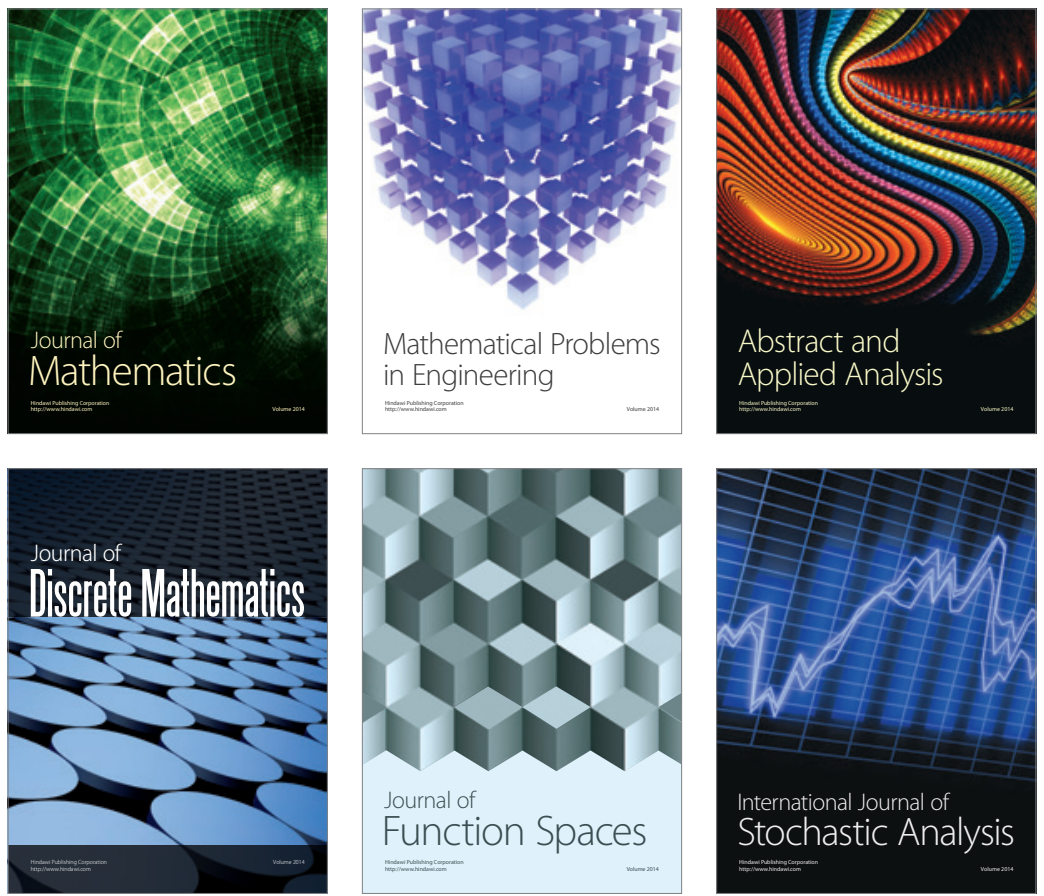

Journal of

Function Spaces

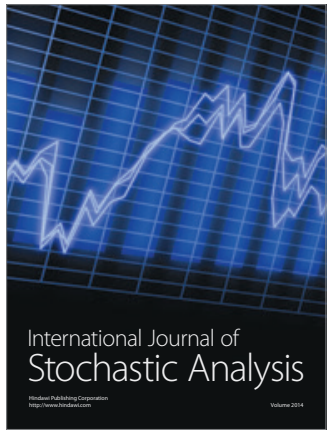

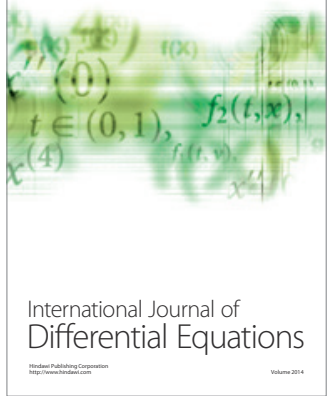
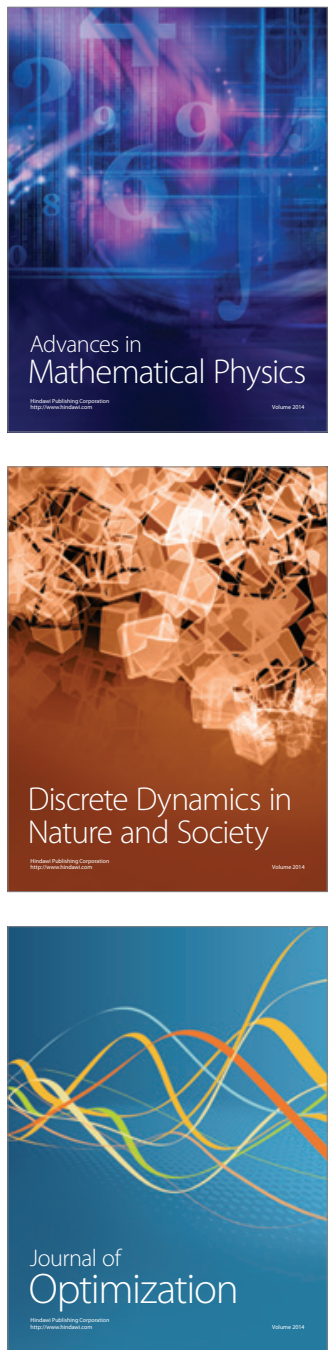